\title{
Diálogos filosóficos. Un enfoque entre la difusión y la divulgación desde de la filosofía de la tecnología.
}

Philosophical dialogues, a approach bettween difussion and divulgation from philosophy of tecnology

Diana Lizbeth Ruiz Rincón

Universidad Autónoma de Chiapas (MÉXICO)

CE: dianaruizrincon@gmail.com / ID ORCID: 0000-0002-0612-0332

DOI: $10.32870 /$ sincronia.axxiv.n77.7a20

Esta obra está bajo una Licencia Creative Commons Atribución-NoComercial 4.0 Internacional $\mathrm{BY} \cdot \mathrm{NC}$

Recibido: 20/08/2019

Revisado: 04/09/2019

Aprobado: 07/10/2019

\section{RESUMEN}

Éste documento busca discurrir entorno a la construcción de un proyecto institucional titulado: "Voces para la filosofía. Diálogos de la academia filosófica contemporánea", que tuvo como objetivo general, el integrar material para el análisis del estado del arte de la filosofía en el contexto externo al estado de Chiapas.

El ejercicio comenzó con miras a la atención a la vida académica, pero pronto se trasladó al escenario de lo social, donde a través del uso de las tecnologías ha sido posible su socialización. Permitiendo así enfatizar de manera concluyente que, la transferencia de tecnologías y la construcción de conocimiento en los marcos de las diversas Líneas de Generación y Aplicación del Conocimiento, requieren de la participación e inclusión de las Tecnologías de la Información y la Comunicación (TIC), pero desde un enfoque crítico y reflexivo.

Una indagatoria respecto a los temas contemporáneos en el campo de la filosofía, no puede dejar fuera los temas tecnológicos ni el uso de los mismos, como recurso tanto problematizador 
como posibilitador; por ello la filosofía de la tecnología como campo de reflexión y estudio filosófico se transfigura en una línea ineludible que atraviesa la actividad humana, así como las consecuencias del uso dependiente de las tecnologías (electrónicas en su mayoría), y que ha acompañado a la humanidad y a la reflexión filosófica desde el nacimiento del hombre como homo sapiens; desde su tendencia al uso, construcción y adaptación de herramientas para enfrentar, entre otras cosas, hasta alcanzar su vida natural y a la naturaleza.

Palabras clave: Difusión. Divulgación. Tecnología. Filosofía de la Tecnología. Redes Sociales.

\section{ABSTRACT}

This paper seeks to reason around the construction of an institutional project entitled: "Voices for philosophy. Dialogues of the contemporary philosophical academy", which had as its general objective, the integrated material for the analysis of the state of the art of philosophy in the context external to the state of Chiapas.

The exercise began with a view to the attention to academic life, but soon moved to the social scene, where through the use of technologies it has been possible to socialize. Thus allowing to emphasize conclusively that, the transfer of technologies and the construction of knowledge within the framework of the various Lines of Generation and Application of Knowledge, difficulties of participation and inclusion of Information and Communication Technologies (ICT), but from a critical and reflexive approach.

An inquiry regarding contemporary issues in the field of philosophy, can't leave out the technological issues or the use of them, as a resource both problematizer and enabler; that is why the philosophy of technology as a field of reflection and philosophical study is transfigured in an inescapable line that crosses human activity, as well as the consequences of the dependent use of technologies (mostly electronic), and that has accompanied humanity and to philosophical reflection from the birth of man as homo sapiens; from its tendency to the use, construction and adaptation of tools to face, among other things, to reach its natural life and nature.

Keywords: Difussion. Divulgation. Technology. Technology of Philosophi. Social Networks. 


\section{Introducción}

El proyecto de investigación Voces para la filosofía. Diálogos de la academia filosófica contemporánea, registrado ante la Dirección General de Investigación y Posgrado de la Universidad Autónoma de Chiapas, con recursos propios, busca integrar material para el análisis del estado del arte de la filosofía en el contexto externo al estado de Chiapas. Para ello se llevó a cabo una convocatoria a cada una de las Instituciones de Educación Superior en México donde se oferta la Licenciatura en Filosofía, extendiendo dicha invitación a la Universidad de Salamanca y la Universidad de León, ambas instituciones españolas; de modo que, a través de la impartición de una serie de Conferencias Magistrales con tópicos que permitieran incorporar la fundamentación teórica de manera reflexiva al conjunto de conocimientos de especialistas en un sentido amplio.

Partiendo de lo anterior, la siguiente exposición se organiza de la siguiente manera: en el primer apartado realizamos una exposición sintética de los espacios en donde se fomentan las actividades de difusión del conocimiento (filosófico), estas son las instituciones de educación superior; ello como preludio para la presentación del proyecto de investigación previamente referido, que constituirá el segundo apartado, y cuyo origen se sitúa precisamente como una acción para la difusión de la filosofía. En el transcurrir de las actividades del dicho proyecto, que son narradas en el tercer apartado, es posible apreciar ciertas tensiones entre la perspectiva tradicional de los contenidos sobre la enseñanza/aprendizaje de la filosofía, y los contenidos emergentes que se hayan en una etapa de germen o florecimiento reciente en las que se había asumido académicamente la socialización del conocimiento; sin embargo dichas tensiones se asumen como no negativas en tanto se hace patente la participación de las tecnologías, tema que integra el cuarto apartado, y del cual puede reflexionarse filosóficamente; por lo que ésta perspectiva filosófica nos permitirá generar un encuentro entre las actividades o acciones de difusión y divulgación del conocimiento filosófico. 


\section{La actividad académica como parangón de la difusión filosófica}

En el campo de la filosofía resulta más que claro que para sostener alguna opinión o creencia, es necesario contar con un cuerpo de afirmaciones que acompañen y fortalezcan la idea central, y que dichas afirmaciones deben de venir acompañadas de cierta estructura, orden y claridad, que permitan no solo exponer a otros, dicho contenido, sino persuadir respecto a su relevancia, consistencia, legitimidad o inclusive veracidad.

De esta manera, la premisa que se pretende exponer en esta primera sección, corresponde con intentar representar como correspondientes la noción de difusión filosófica con lo que conocemos propiamente como actividad académica, principalmente en las Instituciones de Educación Superior (IES). Es decir, habremos de presentar sucintamente las condiciones institucionales que motivaron la construcción de un proyecto denominado Voces para la Filosofía. Diálogos de la academia filosófica contemporánea, tema vertebral de este trabajo.

Desde una visión no solo retrospectiva sino a su vez introspectiva de los procesos formativos del programa académico de la Licenciatura en Filosofía de la Universidad Autónoma de Chiapas (UNACH-México), nos colocarnos en un contexto emergente de formación filosófica en el que nos encontramos con una ausencia de tradición, lo que no es negativo en todo sentido, pues permite a los agentes involucrados el ir surcando caminos y líneas de investigación, al tiempo vincularse en el acompañamiento con otros colegas en los caminos de la filosofía y la formación de profesionales en éste campo del conocimiento humano.

Más allá de intentar seguir el hilo de las investigaciones que de modo puntual y exhaustivo se realizan en los márgenes de estos contenidos, lo que se busca es exponer las condiciones desde las que un programa de reciente creación y con la finalidad de contar la legitimación y convalidación de los espacios que desde la institucionalidad se han organizado para acuerpar y acompañar a este tipo de programas que pertenecen a las humanidades. Así, la Licenciatura en Filosofía de la UNACH, en el año 2016 comenzó los procesos para su evaluación y acreditación.

Éste programa comienza con la valoración respecto a su pertinencia en la región en la que se encuentra inmerso. Para atender adecuadamente las exigencias que el contexto mexicano demanda 
a la actividad filosófica se ha sido necesario establecer el contacto con las políticas educativas, con la sociedad, el mercado laboral, con el Sistema Educativo Mexicano, con las culturas y con los agentes educativos. Además, ha de tenerse en cuenta las reformas educativas en México, a las cuales el Plan de Estudios la Licenciatura en Filosofía de la UNACH ha prestado una atención especial a través de la línea de Docencia Filosófica; sin embargo una evaluación atenta indicará las acciones a ejecutar tomando en cuenta los constantes cambios educativos ya dados o los que están en operación y los que están en ciernes para mantener esa pertinencia, en especial en el Nivel Medio Superior, como potencial campo laboral del egresado en Filosofía en el estado de Chiapas.

No obstante, ese no es el único campo laboral del filósofo. Por ello, ha de considerarse en particular la eficacia de la escuela para la consecución de habilidades relevantes, cuestión que deberá ser tomada en cuenta por las políticas educativas. Esto atañe o afecta, desde luego, a cualquier disciplina. Por lo que estas habilidades relevantes tienen que ser claramente expuestas y justificadas en el curriculum de filosofía (y no únicamente en las políticas educativas) para plantear con claridad y de manera definitiva el papel del filósofo en la sociedad.

De esta manera, y como uno de los aspectos de mayor relevancia, resultó el abrir y promover el diálogo hacia otras fronteras a través de investigadores de otras IES nacionales e inclusive internacionales, que permitieran el arribo de los temas de mayor repunte en mundo de la filosofía.

Con lo anterior, lo que se busca es contar con un estudio cuyos resultados provean claridad respecto a la pertinencia social de la Licenciatura en Filosofía de manera actualizada y vigente, que abone a las actividades de reestructuración del Plan de Estudios, pero sobretodo que abra los escenarios para el ejercicio dialógico del cultivo de la filosofía. Debido a que se ha observado la necesidad de actualizar/reestructurar el Plan de Estudios de la Licenciatura en Filosofía. Por tal motivo, es necesario iniciar los trabajos de rediseño curricular, a partir de los resultados que el estudio de pertinencia arroje.

Los sitios institucionales, los contextos académicos, las universidades pues, son los grandes escenarios sobre los que se han construido diversos modos de mantener la vigencia filosófica, no 
por su impronta relevancia o contenido, si no por los olvidos a los que se le somete en función de la "disponibilidad de las autoridades". Los espacios privilegiados para la difusión de la filosofía son los espacios académicos, los diversos currículums que forman a los futuros profesionales de la filosofía, a quienes se les encomendará a su vez el mantener encendido el fuego del conocimiento filosófico.

La difusión será pues en este texto, entendida como el acto de compartir información en un contexto y hacia un auditorio que comparte las mismas bases epistémicas sobre la que los discursos son construidos. La difusión se abre a tecnicismos, a términos rebuscados, a la exposición de ideas abstractas y "chistes locales" cuyo significado es compartido por la comunidad epistémica en la que se encuentra.

Los procesos de formación filosófica a nivel de pre-grado, el currículum, el Plan de Estudios, las actividades académicas y extra-curriculares, todo ello formará parte de lo que será entendido como difusión de la filosofía. Los diálogos entre colegas docentes, estudiantes, congéneres de otras IES en congresos, encuentros u otras actividades que se hallen en el universo de la vida académica, se encontraran inmersos en la dinámica interna de éste concepto.

Pero al ser un encuentro entre iguales, entre colegas o hablantes de una misma lengua, entre habitantes de un mismo pueblo, e inclusive entre habitantes de un mismo hogar; el dialogar con otros nos permite ampliar los horizontes de comprensión, nos permite conocernos a nosotros mismos como diría Sócrates. Pues no es sino a través del diálogo que el ejercicio dialéctico permite generar nuevos conocimientos. En este sentido, la demanda interna, esto es, el requerimiento de contar con un acercamiento a los temas de interés de otros espacios formativos en el campo de la filosofía, así como la demanda externa, que consiste en la vinculación con otros contextos que permitan, por un lado, fortaleces los procesos locales, y por otro, el intercambiar ideas y temas.

A raíz de esas circunstancias, se desarrolló el proyecto aludido párrafos arriba: Voces de la Filosofía. Diálogos de la academia filosófica contemporánea. Para lo cual, desde la Coordinación de la Licenciatura en Filosofía de la UNACH, en abril del 2018, solo un par de meses después de haber recibido la constancia como "Programa Acreditado" (y las respectivas recomendaciones), por parte del Consejo para la Acreditación de Programas Educativos en Humanidades (COAPEHUM); se 
hicieron llegar invitaciones por escrito y enviadas vía correo tradicional y electrónico a diversas IES con programas de Licenciatura en Filosofía para que se hiciera extensiva la invitación a los profesores investigadores de sus respectivos programas para participar como parte de las Voces que se presentarían ante el quorum integrado por un público símil, receptor de la difusión de las Conferencias Magistrales.

Este ejercicio de difusión de la filosofía, optó por desarrollarse con ayuda de los recursos tecnológicos que permitieran aprovechar la conectividad para no depender de la negativa del uso de recursos económicos inexistentes. El apelar entonces, a una reflexión desde la filosofía de la tecnología, se realiza pues con el objeto de establecer ciertas nociones que nos permitan transitar con naturalidad de la idea del soporte material al soporte tecnológico como posibilitador de la expansión de un sistema que en el caso del tema que nos ocupa, no solo se queda en el campo de un auditorio con el que se comparten las bases epistémicas, sino que nos llevará a ampliar los discursos hacia otros auditorios.

Entonces, un proyecto que inicia con pretensiones de difusión de la filosofía, se encuentra en una encrucijada que le permitirá, o bien subsumirse a la localidad, o ampliarse a lo transfronterizo, con el apoyo de las tecnologías que, de manera irreflexiva, algunos podrían decir que han condenado a la filosofía.

\section{Voces para la filosofía: El proyecto eyectado}

Diseñar una metodología que permitiera concretar las aspiraciones que el objetivo delineaba, conllevó necesariamente a reconocer de manera inicial dos condiciones: I) la ausencia de recursos económicos que permitieran subsanar los gastos generados por los viáticos de los conferencistas a invitar, y II) la distancia entre el centro y norte del país donde se concentran la mayor parte de programas de Licenciatura en Filosofía; sin mencionar que dichas condiciones se vuelven críticas al tratarse de posibles invitados internacionales.

De esta manera se optó por considerar realizar las presentaciones de las Conferencias Magistrales de manera virtual con el apoyo de las tecnologías, pero antes de ello, se volvía 
menester organizar su desarrollo a partir de los objetivos particulares de éste proyecto. Tomando en cuenta el calendario de clases, y atendiendo a la naturaleza del currículum de la Licenciatura en Filosofía de la UNACH, donde la mayor cantidad de cargas horarias de clases se concentran en el horario vespertino de lunes a jueves, se volvía posible utilizar el último día hábil para éstas actividades. Así, el primer objetivo particular consistió en invitar y calendarizar la participación de 16 expertos en tópicos contemporáneos en el campo de la filosofía. Ello se llevó a cabo a través de invitaciones giradas a los responsables de cada programa en las diversas IES del país, así como a través de invitaciones formalizadas con investigadores internacionales con quienes ya se guardaba cierto contacto.

Las invitaciones se redactaron para ser compartidas con las academias y cuerpos docentes integrados preferentemente por profesionales de la filosofía, cuyo interés y disposición les permitiera por un lado aceptar la invitación, y por el otro, realizar una propuesta temática sobre el contenido de la Conferencia Magistral a dictar. En éste sentido se configuró el segundo objetivo específico, que buscó dictaminar la propuesta de cada Conferencia Magistral en relación a la organización que en puntos posteriores se desarrollará. Así, desde abril y hasta noviembre de 2018, se recepcionaron las confirmaciones, temáticas y se dio inicio al cumplimiento del tercer objetivo particular: calendarizar la presentación de cada una de las Conferencias Magistrales (tomando en cuenta que pudieran ser canceladas en ausencia de seguimiento del Conferencista Magistral invitado).Esto es, reconociendo la dinámica particular de éste ejercicio de inicio académico, en el que los trabajos de difusión de la filosofía, no serían desarrollados como tradicional y “presencialmente" se desarrollan, en espacios físicos que propicien el ejercicio dialógico en torno a los temas presentados; sino que se trataba de la incursión de la virtualidad en las dinámicas íntimas áulicas en espacios de formación en educación superior, tratándose especialmente de temas filosóficos. A razón de ello, era natural esperar que a la distancia se sumara el olvido, y con ello la adquisición de otros compromisos que al demandar mayor "presencialidad" impedirían a nuestros expertos concretar su participación. 
Sin más, con la intensión de no dejarlo todo en manos de lo virtual o intangible, se informó mediante las mismas misivas que, cada discurso dictado en las inmediaciones de las Conferencias Magistrales, deberían ser plasmadas en documentos en extenso en donde cada experto pudiera incorporar, si así lo desease, los aspectos más relevantes del ejercicio dialógico de su presentación. Pues, uno de los aspectos más novedosos de ésta propuesta para la difusión de la filosofía fue la que denominaremos como micrófono abierto. Esto es, la viabilidad para interactuar en tiempo, a través de las redes, entre expositores y auditorios, para el intercambio de ideas, la discusión puntual de ciertos tópicos e inclusive la presentación de ciertas controversias. La idea de micrófono abierto se suscitó debido a la inquietud constante por parte de asistentes y conferencistas respecto a ¿cómo se dialogará con el otro?, esto es, ¿cómo le escucharé y sabré que me escucha?, pues estas disposiciones comunicacionales son las que nos permiten construir un tipo de discurso u otro.

Por último, respecto a los objetivos específicos, y siguiendo la idea del párrafo anterior, se publicará la compilación en extenso de las Conferencias Magistrales en un formato de libro electrónico. Para con ello consolidar la organización y presentación tangible de las miradas a los temas contemporáneos de la academia filosófica, aunque baste aclarar que éste proyecto no agota ni la intensión ni la extensión de los profundos temas en el campo de la filosofía, pero lo que sí hace es reconocer como punto de análisis estos otros temas que se salen del caudal que las tradiciones han surcado a través de la actividad académica y su paso por la historia.

Éstas acciones se derivan de los resultados de la evaluación para la acreditación del Consejo para la Acreditación de Programas en Humanidades (COAPEHUM) al que fuese sometido el Programa de la Licenciatura en Filosofía de la Universidad Autónoma de Chiapas, y cuyas recomendaciones se centran en la restructuración del Plan de Estudios, por lo que fue necesario organizar en dos partes las temáticas a abordar, a saber: I) Disciplinas Filosóficas y II): Ejercicio de la filosofía.

Del desarrollo, avances y resultados preliminares de dicho proyecto, hablaremos enseguida. 


\section{Tensión entre la filosofía tradicional y emergente}

Construir un programa académico que no solo recupere los principios fundamentales que todo profesional de la filosofía debe conocer, sino que, además se presente como una propuesta que se justifique a sí misma en un contexto en el que las humanidades en general y la filosofía en particular, se hallaban en un cuello de botella curricular que, desde la Reforma Educativa que tuvo lugar en el sexenio de Felipe Calderón Hinojosa habían sido condenadas a la transversalidad, por no decir, a su desaparición; y particularmente en uno de los estados con mayores índices de pobreza y marginación, el desafío de abrir el programa de la Licenciatura en Filosofía en una Institución de Educación Superior (IES) pública como la Universidad Autónoma de Chiapas, aumentaba la complejidad, pero no la imposibilidad.

Su propuesta de Plan de Estudios se erige entonces de modo tripartita e inicia con una formación básica cuya duración se ubica en los primeros cuatro semestres de formación, y su perfil estaría consolidándose en cualquiera de las tres líneas de especialización: I) estudios sobre filosofía mexicana, II) lugotutoría ${ }^{1}$ y III) docencia filosófica. No se trata de exponer las particularidades de éste programa, si no de situar al lector entorno a las tensiones dadas desde su origen en torno a la tradición y la emergencia. Situaciones que promoverían este tipo de acciones y por lo cual era imperativo contar con las perspectivas externas, siempre en diálogo con las condiciones internas; así la primera división temática fue: I) Disciplinas Filosóficas y II) Ejercicio de la filosofía.

A partir de la primera división se hizo necesario seccionar, buscando presentar de manera temática, los principales contenidos que todo profesional de la filosofía debe conocer a lo largo de su proceso formativo, y cuya relevancia permitiría hacer pertinente su presentación de la siguiente manera: para la primera sección, de la parte denominada Disciplinas Filosóficas se propuso: a) Historia de la Filosofía y Filosofía Mexicana, dichas líneas propuestas inquirían en relación a temas contemporáneos sobre la enseñanza de la historia de la filosofía, proyectando entre sus tópicos, temas sobre filosofía mexicana o latinoamericana. La sección b) buscó agrupar temas sobre

\footnotetext{
${ }^{1}$ Se refiere a la Logoterapia (fundada por el neurólogo y psiquiatra austriaco Viktor Frankl), pero que al ser de corte clínico impedía su permanencia en un programa de formación filosófica, por lo que se optó por su modificación.
} 
Métodos de la filosofía, en los cuales los investigadores participantes pudieran encontrar el espacio idóneo para hablar en relación a las perspectivas o posicionamientos más actuales.

La tercera sección c), centró su temática en torno a la Filosofía aplicada. A partir de ésta sección, la organización apuntalaba a una invitación a tensar las formas tradicionales (principalmente de corte historicista, parte de la herencia hegeliana) de aprender/enseñar filosofía, respecto de las formas emergentes, en donde la filosofía no se ve más como solo actividad contemplativa y especulativa, sino donde la transmutabilidad de las formas teóricas a formas prácticas ha llevado a explorar e innovar en otros derroteros de aplicabilidad a la filosofía en sus amplísimas disciplinas.

Así, la última sección que integraría ésta primera parte, la sección d), tocaría temas relacionados con las "Prácticas Filosóficas, filosofía para niños, filosofía y educación"; ésta cuarta sección es un banquete al que se sumarían más investigadores de los programados, esto es, por orden organizativo y coherencia en la exposición de las ideas, se ofertaron espacios para dos investigadores por sección; empero, para ésta última sección, fue el doble de expertos en el campo quienes aceptaron la invitación para dictar una conferencia magistral.

La segunda parte del ciclo de conferencias magistrales abordaron temas en torno al Ejercicio de la filosofía. Recordemos que, el público principal receptor de los contenidos del proyecto Voces para la Filosofía. Diálogos con la academia filosófica contemporánea se hallaba en la comunidad del programa académico de la Licenciatura en Filosofía de la Universidad Autónoma de Chiapas: estudiantes, docentes, investigadores y autoridades administrativas; por ello, dialogar en torno al Ejercicio de la filosofía, se constituyó como una forma de escudriñar respecto a las condiciones que enfrentará el profesional de la filosofía más allá del contexto áulico y académico. Las secciones que se abrieron para dichos fines fueron: e) Campo laboral, nuevas fronteras; f) El profesional de la filosofía en distintos niveles educativos; g) Representaciones sociales de la filosofía; y h) La filosofía más allá de occidente.

Logra observarse que cada una de estas temáticas buscan hacer converger diálogos alrededor de aspectos desde los que se relaciona el ejercicio profesional de la filosofía. Al respecto, 
se destacan no solamente tópicos o prácticas investigativas, sino la pertinencia del profesional de la filosofía en el momento histórico actual de nuestro país, en donde mediante el esfuerzo de consolidados filósofos e investigadores de la filosofía se ha llevado a nivel de propuesta la inclusión de contenidos filosóficos en todos los niveles y subsistemas del sistema educativo mexicano; allí pues, se vuelve imperativa la participación de egresados de éstos programas.

Aunado a ello, el dialogo sobre las representaciones sociales de la filosofía se proponía como un ejercicio necesario para promover los vínculos entre la academia (filosófica) y la sociedad propia de los contextos en los que tiene lugar. Sin embargo, las dos últimas dos secciones $g$ ) y $h$ ), no encontraron el eco suficiente para su tratamiento, por lo que los espacios quedaron prácticamente desiertos.

Resultó notoria una tensión no rupturista entre las reflexiones disciplinares y profesionales de la filosofía en la atención al llamado realizado a investigadores y filósofos de amplia trayectoria en formación. Así, la metodología para la realización del proyecto se concentró en la organización de las presentaciones; se procuró que cada una de las ocho secciones estuviese conformada por dos Conferencias Magistrales que habrían de presentarse a distancia y de manera virtual con una duración mínima 80 minutos y máxima de 100 minutos, dando tiempo suficiente para atender a una ronda de preguntas y comentarios por parte del auditorio, integrado éste en su mayoría, por estudiantes de los primeros semestres de la Licenciatura en Filosofía de la Universidad Autónoma de Chiapas, docentes y algunos invitados ocasionales.

La organización requirió de un seguimiento arduo que permitió se presentaran en el siguiente orden, siendo uno de los recursos constante, los medios digitales de comunicación como el Facebook y los grupos de WhatsApp ${ }^{2}$, desde donde se hacían circular imágenes como la de la figura 1:

\footnotetext{
2 Véase la figura 1, donde se muestra la imagen con la cual se hacía difusión de cada una de las Conferencias Magistrales, con la información respectiva.
} 
Imagen 1. (Voces para la Filosofía Diálogos con el pensamiento, 2019).

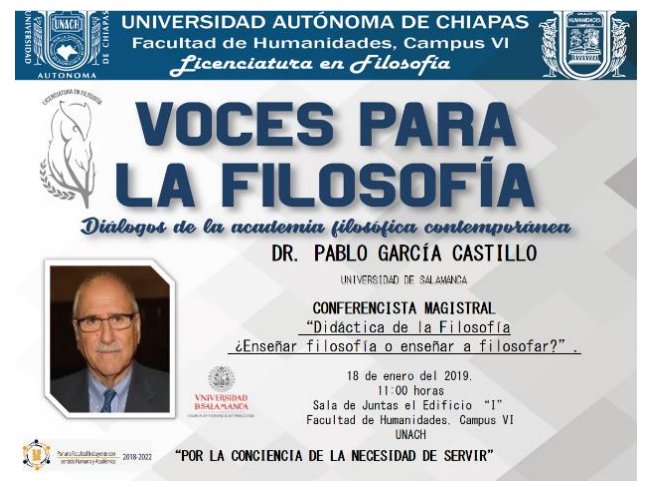

Para la ejecución de las Conferencias Magistrales, fue necesario contar con una Red de Internet que permitiera mantener sin interrupciones las videoconferencias, logrando así la calidad en audio y video para las interacciones entre expertos y el auditorio. De esta manera, contar con la colaboración del estudiante de la Licenciatura en Filosofía que cursaba su último semestre, como soporte tecnológico que facilitó la trasmisión y grabación de las Conferencias Magistrales dictadas.

El proyecto pues, representó una investigación de las investigaciones vigentes en el ámbito de la filosofía que realizan colegas en otros espacios académicos, así como en otras latitudes; permitiendo con ello realizar una meta-investigación filosófica, desde donde buscan exponerse los distintos caminos teóricos y las formas en las que la filosofía práctica va recuperando los espacios que del ejercicio profesional de la filosofía demanda la sociedad.

Sin lugar a dudas, el análisis y la reflexión constante respecto a los caminos y derroteros de formación en los espacios de Educación Superior, no simplemente requieren, sino que demandan la ubicación de intersticios de comunicación que permita tener en cuenta, cualquiera que sea el campo disciplinar de que se trate, cuáles son los temas, las rutas, los caminos, los proyectos, las grandes preguntas que en el campo otros colegas y congéneres, intentan responder. Ya sea para generar Redes, propiciar vínculos, o para tener los elementos que permitan una introspección razonada desde la continuidad o la ruptura de ejercicios formativos similares y que pueden sernos de luz en tiempos de búsqueda. 
De los temas propuestos por cada uno de los expertos en el campo de la filosofía convocados, y relacionado a cada tema marcado en las secciones correspondientes, es posible observar en los títulos presentados guardan una estrecha relación no sólo con los intereses individuales de investigación, sino con una forma diferente de posicionarse frente al ejercicio profesional de la filosofía, que no se trata ya, de una mera trasmisión de conocimientos sin sentido o datos históricos, sino de vincular al futuro profesional de la filosofía con otras formas de realización laboral, pero también otros tópicos de interés para el desarrollo de ulteriores investigaciones.

Programadas los días viernes a partir del 11 de enero y hasta el 03 de mayo de 2019, la serie de Conferencias Magistrales confirmadas fueron quince (15), y los títulos propuestos por sus autores, que incluso, desde una sección disciplinar, marcan y remarcan las relaciones que las distintas ramas o disciplinas filosóficas guardan con el mundo exterior, con el contexto social y el compromiso del profesional de la filosofía, en el que desde las voces de los expertos fue particularmente insistente el invitar a los jóvenes en formación a idear modos distintos de intervenir desde el ámbito laboral a la transformación de las representaciones sociales de la filosofía, marcando de manera constante en cada Conferencia Magistral que no solo la sociedad, la academia, o la realidad en cualquiera de sus formas necesita a la filosofía, sino que la filosofía requiere del mundo externo y sus elementos para realizarse.

Los bloques de las conferencias y la resonancia en torno a la pluralidad de temáticas metodológicas y la escasez de ponentes en torno al ejercicio profesional de la filosofía, parecieran que de manera lineal se da preponderancia nuevamente al aspecto académico más que al social de la filosofía.

\section{Las tecnologías en el proceso de divulgación}

Para nuestro último apartado, requeriremos extrapolar los elementos a los que hemos aludido, pero en esta ocasión de manera sintética, de modo tal que podamos establecer las relaciones entre ellos, y al final con el enfoque que daremos a manera de cierre desde la filosofía de la tecnología. 
Comenzamos con la exposición de la actividad académica en los espacios de educación superior como formas de difusión de la filosofía, entendiendo a la difusión como una actividad generadora de discursos dirigidos a un público que comparte las mismas bases epistémicas; así, el proyecto elucidado nace con el objetivo de difundir en la comunidad académica los temas y problemas contemporáneos que son material de investigación de colegas en contextos diversos, nacionales e internacionales.

En la realización de dicho proyecto se muestra una tensión entre la forma tradicional de enseñar y aprender filosofía, respecto a formas emergentes que responden mayormente a las demandas sociales y contemporáneas. Por ello y para la realización de dicho proyecto, fue necesario incorporar a las Tecnologías de la Información y la Comunicación (TIC), de forma tal que pudieran llevarse a cabo las conferencias magistrales a distancia, pero en una versión 2.0 que implica un intercambio y respuesta entre tecnologías y usuarios, esto es, las plataformas de telecomunicaciones permitieron que expertos y auditorio dialogaran en línea, al tiempo que se generaba el resguardo del ejercicio en dicha plataforma.

El uso de la tecnología hubo de modificar la manera de construir, dirigir y dirimir los discursos para un auditorio específico (en acto) pero más amplio en potencia. Al mantener un historial digital de las ahora videoconferencias magistrales, alteró (no en un sentido negativo) las formas tradicionales en las que se había asumido académicamente la socialización del conocimiento. Ahora la incorporación de las tecnologías a estas prácticas, las trastocan en un sentido divulgativo; estando con ello en posibilidad de llegar a un mayor número de agentes epistémicos, según los usuarios de las plataformas en las que se comparta.

En este sentido, resulta imperante resaltar un par de aspectos que guardan estrecha relación con el uso de las tecnologías y la distinción entre difusión y divulgación del conocimiento (filosófico). Por un lado, los Conferencistas Magistrales, en su totalidad reconocieron una nula experiencia en la impartición de conferencias a distancia, es decir, contaban experiencias de ponencias dictadas a un auditorio que eran transmitidas en vivo por algún dispositivo electrónico y 
con el auxilio de alguna plataforma tecnológica, pero en donde los asistentes se encontraban presentes y con la posibilidad de interacción directa.

Estos discernimientos forman parte del emergente conjunto de tópicos con los que se relaciona la filosofía de la tecnología. Miguel Ángel Quintanilla (2017) en la presentación a la segunda edición de su texto, señala que: “[...] el mundo que queremos construir no depende tanto de las tecnologías que hoy tenemos disponibles cuanto del tipo de tecnologías que decidamos desarrollar para el futuro" (p. 9). A lo que en el prólogo agrega:

[...] no hay verdadero y sano desarrollo tecnológico sin una adecuada integración de la tecnología en el resto de la cultura, lo cual resulta difícil de llevar a cabo si no es en un ambiente en el que se respete a libertad, se valore el trabajo bien hecho y se incentive la creatividad. (p. 17)

Empero, un ejercicio de difusión, que también podría tener alcances divulgativos ${ }^{3}$, en el que ellas y ellos, en su expertiz, se encontraran solos y frente a un ordenador, en ausencia de un público "directo", expresaran sus ideas e idearan nuevas formas de interacción que, además, con el apoyo de las nuevas tecnologías de la información y la comunicación (como el sitio de YouTube ${ }^{\circledR}$ ) pudieran ser registradas, almacenadas para la posteridad y con ello mayormente divulgadas.

Es decir, dados los contextos desde donde los colegas expertos dictaban sus Conferencias Magistrales, resultó necesario abrir une canal de YouTube desde el cual fuera posible compartir "en vivo" dichos ejercicios, y que se denominó: Voces para la filosofía. Diálogos con el pensamiento, la como muestra la figura No. 2 Canal de YouTube:

\footnotetext{
3 Entenderemos por difusión, el socializar algún contenido en un contexto especializado; mientras que la divulgación buscará socializar dicho contenido a un público más amplio y no especializado.
} 
Imágen 2. Canal de YouTube (Voces para la Filosofía Diálogos con el pensamiento, 2019).

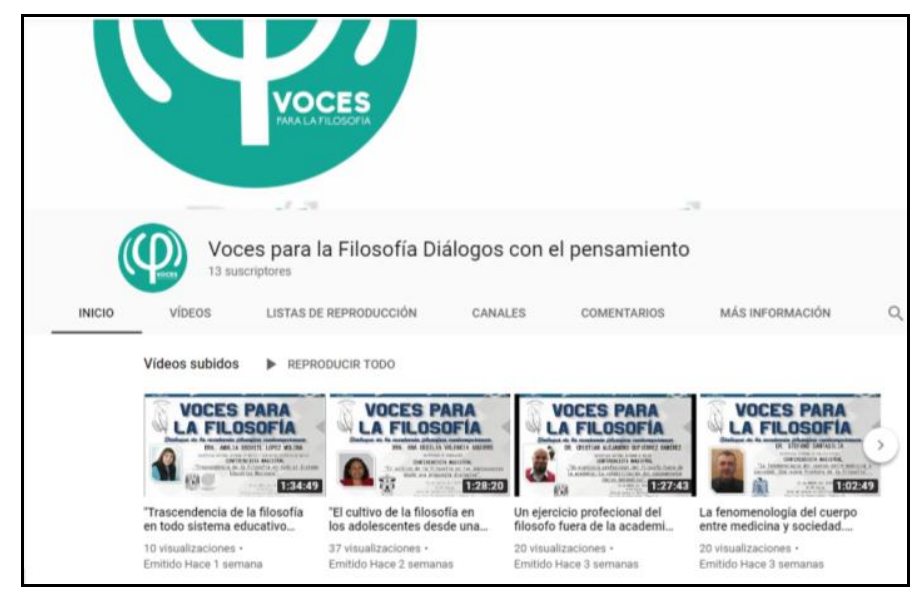

El trasladar de un tradicional escenario físico y tangible, a las actividades académicas a las que estamos habituados a congregarnos en pequeños grupos, ésta plataforma tecnológica permite transitar entonces del soporte material o de la materialidad, a un soporte digital. A este respecto nuestro autor agrega:

El conglomerado de nuevas tecnologías, son su implacable invasión en todos los ámbitos de la vida humana, supone una configuración de la técnica completamente nueva en la historia de la humanidad. Nunca como hasta ahora había estado la sociedad en su conjunto tan articulada en torno a la actividad tecnológica, y nunca la tecnología había tenido tan fuertes repercusiones sobre la estructura social, y en especial la estructura cultural de una sociedad. (Quintanilla, 2017, pág. 30).

Si en el existencialismo la máxima sartreana reza que "el hombre es lo que hace con lo que hicieron de él", para el campo de estudio que atañe a la reflexión filosófica de la tecnología, podríamos decir que: "la tecnología será lo que el ser humano decida en su accionar".

En definitiva, pues, la tecnología actual tiene efectos decisivos en los componentes más peculiares de nuestra cultura: nuestros sistemas de conocimientos, nuestras pautas de comportamiento y nuestros sistemas de valores. Y ello no de una forma esporádica y 
accidental, sino de manera sistemática, continua, intensa y general. (Quintanilla, 2017, pág. 31).

De esta manera resulta más natural establecer el enfoque entre la difusión y la divulgación desde la filosofía de la tecnología. Pues a partir del proyecto aludido, convergen los mecanismos por antonomasia para la difusión de la filosofía en contextos académicos en donde primara el encuentro frente a frente; sin embargo, y aunque las tecnologías no excluyen en lo más mínimo éste tipo de elementos, lo que sí permiten en florecimiento de mecanismos para la misma divulgación. Lo anterior se retrata de modo más sencillo señalando que, cada uno de los investigadores participantes, manifestaron, independientemente de la temática a la que se hallen adheridos, la novedad en dicho formato de participación. Esto es, el "modificar los sistemas" desde los cuales se está habituado a desarrollar la exposición de un conjunto de ideas ante un auditorio que nos lee y al cual leemos en su gesticulación afirmativa o recriminatoria, para apuntalar énfasis o ajustes en nuestros discursos, se desdibujan al mediar la tecnología; entonces el autor se encuentra frente a sí mismo y la conciencia de un discurso que sabe que una vez generado, permanecerá de manera cuasi perenne en los océanos del ciberespacio.

En este contexto de acciones es donde se vuelve imprescindible la participación axiológica y epistemológica de la filosofía, como guía problematizadora a cuyo encuentro deben aproximarse campos del conocimiento humano que de alguna u otra manera se relacionen con la tecnología (la técnica no queda relegada, por cierto), so pretexto de los cambios culturales generados, las innovaciones motivadas, los productos esperados e inesperados y los impactos en la generación del conocimiento.

\section{Conclusiones}

A manera de conclusión, podemos iniciar sosteniendo que pese a lo que se asume como la firmeza de la tradición, fue posible distinguir que no ha sido viable si no desde la autocrítica y la reflexión razonada, que la tradición debe venir acompañada de rupturas que permitan su desarrollo y 
evolución. Que un campo que no se cuestione a sí mismo y un proyecto que no escuche otras voces, se condena a luchar en los marcos de la justificación sin más soporte que su propia ceguera.

La distinción entre lo natural y lo artificial resulta tan innecesaria como la que se realiza entre nóumeno y fenómeno. Se tratan de elementos, procesos, fases, etapas, etc., que en su devenir dan lugar a aquello que experimentamos como agentes epistémicos. La filosofía no puede estar separada de algún tipo de soporte material, ya sean los mismos seres humanos, con su oralidad; o los productos de la humanidad, como los libros o los recursos electrónicos. La filosofía es provocadora (mucho más que provocativa) y en este sentido debe (en la acepción que todo imperativo categórico impele) asirse de cualquier soporte para elevar la vista y hacer llegar hasta la distancia más lejana un juicio que lo haga temblar todo.

Los retos de los sujetos epistémicos frente a la vorágine de información, supone el tratamiento y construcción de criterios que busquen no solo normar sus relaciones, sino que transgredan a las mismas procurando con ello no despegar el quehacer filosófico, del más elemental al más elevado, del contexto al cual debe interrogar y a quien debe a su vez hacer llegar los esfuerzos por responder sus grandes interrogantes. El proyecto: Voces para la filosofía. Diálogos de la academia filosófica contemporánea, alcanza sus objetivos gracias al esfuerzo y participación de Ángel Enrique Castro Vázquez, estudiante de la Licenciatura en Filosofía de la Universidad Autónoma de Chiapas, quien por sus conocimientos en el ámbito de la tecnología y su tema de tesis coincidimos en éste interés.

De esta manera, los resultados no concluyen únicamente con la videoconferencia magistral realizada de manera virtual, nuestros autores invitados han enviado el documento en extenso de los temas expuestos una vez realizada una minuciosa revisión y adecuación, producto del intercambio realizado a distancia. De esta forma, será posible también publicar en su versión digital, el libro producto de éste proyecto, bajo el mismo título.

Finalmente, debemos hacer hincapié en que el ejercicio de formación colectiva que fuera organizado en torno al encuentro de diversas perspectivas y enfoques contemporáneos relacionados con la filosofía y su ejercicio profesional, cuyo medio de realización fueron 
prominentemente las tecnologías, nos han permitido hacer dialogar a partir de dos nociones que, desde el ámbito de lo tecnológico e informático, adquieren una relevancia inusitada. Tanto la difusión como la divulgación encuentran espacios de proyección sobre todo a través de las redes sociales, mediante grupos tan especializados o tan abiertos que resultaría sumamente fácil perderse; y aquello que iniciaba con la finalidad de hacer difusión en un espacio local respecto a los temas que en el exterior se priorizaban en el campo de la filosofía, resultó ser más provocativo de un ejercicio divulgativo mediado por las tecnologías. La ciber-cultura, las modificaciones de las relaciones sociales y la organización cultural son temas que requieren de la óptica crítica que están en condiciones de aportar hoy en día los filósofos de la tecnología.

\section{Referencias}

Quintanilla, M. Á. (2017). Tecnología: un enfoque filosófico y otros ensayos de filosofía de la tecnología. Ciudad de México: FCE.

Voces para la Filosofía, Diálogos con el pensamiento. (10 de marzo de 2019). Didáctica de la filosofía ¿enseñar filosofía o enseñar a pensar, Dr. Pablo Castillo García. CM 1. [Archivo de video]. Recuperado de https://www.youtube.com/watch?v=EZa9uVDXGpU\&t=709s

Voces para la Filosofía Diálogos con el pensamiento. (11 de enero de 2019). Voces para la Filosofía

Diálogos con el pensamiento. [Sitio web]. Recuperado de https://www.youtube.com/channel/UCaHCxperESGukmDbxT2Yg-w/featured. 